\title{
Lipophilisation enzymatique de composés phénoliques et évaluation de leurs propriétés antioxydantes
}

Luis Javier LOPEZ GIRALDO

La biomasse végétale, et plus particulièrement celle issue des pays du Sud, suscite un intérêt grandissant en raison de la très large gamme de molécules bioactives qu'elle renferme. Parmi ces molécules, les composés phénoliques semblent particulièrement prometteurs, de par leur ubiquité dans le règne végétal, mais également pour les fortes propriétés antioxydantes, antimicrobiennes, antivirales et anticancérigènes qu'ils présentent. Ces composés s'avèrent, par conséquent, de très bons candidats en tant qu'agents de conservation dans les industries agroalimentaires et cosmétiques, mais également en tant qu'agents thérapeutiques.

\begin{abstract}
The oxidation of unsaturated lipids, which is one of the major causes of foods and cosmetics adulteration, may be limited by the use of antioxidants. Nowadays, phenolic compounds (particularly phenolic acids and flavonoids) generate a growing interest owing to their antioxidant properties. However, the implementation of such polar molecules in lipid-based systems (emulsion or other) is difficult and can lead to a decrease of their efficiency. To solve this issue, one strategy consists in adjusting the polarity of these molecules by the grafting of aliphatic chains of different lengths. The first part of this work has been dedicated to the enzymatic synthesis of a series of chlorogenate and rosmarinate alkyl esters. Regarding chlorogenic acid, the best results were obtained using a two-steps strategy. This strategy involves a preliminary esterification with methanol in presence of a sulfonic resin followed by an enzymatic transesterification (Candida antarctica B lipase) of methyl chlorogenate, with alcohols of 4-16 carbon chain lengths. Under optimal conditions, the initial rates of transesterification were up to two-fold higher than that of direct enzymatic esterification. Furthermore, the two-steps strategy led to overall yields of $61 \%$ to $93 \%$ against $40 \%$ to $60 \%$ in the case of direct esterification. With regard to rosmarinic acid, only the direct esterification in the presence of sulfonic resin leads to satisfactory yields (82\% to 99\%, depending on alkyl chain length). In the second part of this work, we evaluated, from kinetic and stationary point of view, the impact of hydrophobation on the ability of new molecules to scavenge the stable radical 2,2-diphenyl-1-picrylhydrazyl (DPPH $\left.{ }^{\circ}\right)$. The kinetic data showed that chlorogenic acid was stabilized in its quinone form, while esters tend to form dimers from their semi-quinone form. The results obtained at stationary state indicated that both acids, but also their corresponding esters, have a radical scavenging activity greater than the theoretical capacity attributable to the number of phenolic hydroxyl. These results can be explained considering the existence of regeneration and/ or dimerization mechanisms. In the third part of this work the antioxidant capacity of lipophilic derivatives of chlorogenic and rosmarinic acids was evaluated in emulsified systems by a new methodology called conjugated autoxidizable triene (CAT). The results showed that the increase of alkyl chain length did not necessarily lead to an improvement in the antioxidant capacity as predicted the so called "Polar Paradox". Indeed, maximum antioxidant capacities for chain length of 12 and 8 carbon atoms were observed for fatty esters of chlorogenic and rosmarinic acid, respectively. The main hypotheses proposed to explain this unexpected behaviour were based on the distribution of antioxidants and their oxidized forms in the different phase of the system.
\end{abstract}

Key words: phenolic acids, lipophilisation, enzymatic, antioxidant, heterogeneous medium

Parmi les composés phénoliques présents dans le règne végétal nous nous sommes plus particulièrement intéressés aux acides chlorogénique et rosmarinique, présents en grandes quantités dans les extraits de café vert et de romarin. Cependant, malgré leurs potentialités, la mise en œuvre et l'efficacité de ces molécules dans les matrices lipidiques, et plus particulièrement dans les émulsions, se trouvent limitées en raison de leur forte polarité. Pour pallier ces limitations, l'une des stratégies consiste à diminuer leur polarité par le greffage d'une chaîne aliphatique de longueur variable (via une réaction de transfert d'acyles). Ces réactions d'hydrophobation, également appelées réaction de lipophilisation, peuvent être réalisées par catalyse chimique (acide ou basique) ou enzymatique (lipase). Bien que ces deux types de réaction présentent des avantages et des inconvénients, l'intérêt supérieur des lipases est de pouvoir mettre en œuvre des réactions d'estérification sélectives et dans des conditions douces de température qui préserve les composés phénoliques de toute dégradation thermique. De plus, dans un contexte réglementaire favorable au développement de procédés plus respectueux de l'environnement, la catalyse enzymatique apparait comme une alternative intéressante. 
Ainsi, dans la première partie de ce travail de thèse, nous nous sommes particulièrement intéressés à l'optimisation des paramètres de synthèse impliqués dans les réactions de lipophilisation enzymatique d'esters alkyliques $\mathrm{d}^{\prime}$ acides chlorogénique et rosmarinique. À ce sujet, les résultats obtenus ont montré que l'estérification enzymatique directe de l'acide chlorogénique par des alcools gras était beaucoup moins efficace que le transfert d'acyle entre l'alcool et l'ester méthylique de l'acide chlorogénique (ce dernier étant synthétisé par catalyse chimique en présence d'une résine sulfonique). Ainsi, ce procédé mixte, dit chimioenzymatique, de synthèse d'esters lipophilisés $d^{\prime}$ acide chlorogénique, passant par un intermédiaire de type ester méthylique s'est avéré être la meilleure stratégie. En effet, la lipophilisation par transestérification de cet intermédiaire alkyle présente, contrairement à l'estérification directe de l'acide libre, l'avantage de générer un co-produit (méthanol) pouvant être facilement éliminé en continu. De ce fait, l'équilibre chimique est déplacé en faveur de la synthèse à un point tel qui avoisine les conditions d'irréversibilité (disparition d'un co-produit de la réaction). De plus, la valeur d'aw optimale $(0,05)$ déterminée pour l'enzyme, permet de conclure qu'en milieu organique, une faible hydratation de la lipase de Candida antarctica $B$ est absolument nécessaire pour une synthèse performante. Concernant la lipophilisation enzymatique de l'acide rosmarinique ou de son ester méthylique, nous avons constaté que l'utilisation des paramètres précédemment optimisés pour l'acide chlorogénique, conduisaient, dans le meilleur des cas (transestérification), à des rendements avoisinant seulement $8 \%$ après 11 jours de réaction. Une explication à ce comportement inattendu pourrait être l'inhibition de la lipase lors de l'état de transition 1 et nous avons alors postulé que l'encombrement stérique de l'acide rosmarinique en était vraisemblablement la cause. À l'avenir, il serait donc intéressant de valider cette hypothèse en utilisant des conditions réactionnelles spécifiquement optimisées pour la synthèse de dérivés lipophiles $d^{\prime}$ acide rosmarinique. D'autre part, si la modification des paramètres réactionnels ne conduisait pas à une amélioration significative du rendement, l'emploi d'autres lipases pourrait être également envisagé pour la production de dérivés lipophiles d'acide rosmarinique.

Dans un deuxième temps, nous avons étudié les propriétés antiradicalaires des esters ainsi formés. L'influence de la longueur de la chaîne alkyle greffée (1 à 20 atomes de carbone) sur ce type d'activité a plus spécifiquement été examinée. Pour cela, I'emploi du radical 2,2diphényl-1-picrylhydrazyl (DPPH) absorbant dans le spectre visible semble particulièrement
Thèse soutenue le 10 décembre 2008 devant le jury composé de : Patricia Le Bail : INRA Nantes, Rapporteur Michel Girardin : ENSAIA Nancy, Rapporteur

Frédéric Carrière : CNRS Marseille, Membre du jury Gaëlle Pencreac'h: IUT Génie Biologie Laval, Membre du jury

Maria Cruz Figueroa-Espinoza: CIRADSUPAGRO Montpellier, Membre du jury Pierre Villeneuve: CIRAD Montpellier, Directeur de thèse

bien adapté. Dans ce contexte, nous nous sommes intéressés à l'évaluation des capacités antiradicalaires, des points de vue cinétique et stationnaire. Concernant l'acide chlorogénique et ses esters, l'étude cinétique a montré que le mécanisme de transfert d'électron (TE) était la voie réactionnelle la plus probable pour expliquer la stabilisation des radicaux DPPH $\bullet$. De plus, les ordres partiels de réaction obtenus pendant les premières minutes de réaction suggèrent que les voies mécanistiques impliquées sont différentes pour l'acide chlorogénique et ses esters. En effet, pendant les premières minutes de la réaction l'acide chlorogénique peut stabiliser deux molécules de DPPH• contre une seulement pour ses esters. Ces résultats ont été confirmés par CLHP-SM en montrant que, suite à la cession successive de deux atomes d'hydrogène, I'acide chlorogénique se stabilise sous sa forme o-quinone, tandis qu'après la cession d'un atome d'hydrogène ses esters suivent une réaction de dimérisation entre deux radicaux semi-quinones. Concernant les valeurs nes (nombre de molécules de DPPH• réduites par mole d'antioxydant) obtenues à l'état stationnaire, les acides chlorogénique et rosmarinique, mais également leurs esters respectifs, montrent une capacité réductrice supérieure à la capacité théorique imputable à leur seule structure (nombre d'hydroxyles phénolique). Ces résultats peuvent être rationalisés si, outre la structure moléculaire des antioxydants, des réactions entre les produits d'oxydation et le milieu sont considérées. Ainsi, nous avons proposé que les hautes valeurs de nes obtenues pouvaient être expliquées en considérant l'existence de réactions de régénération et/ou de dimérisation. Cependant, bien que ces voies soient en mesure d'expliquer les valeurs de nes, un long travail d'approfondissement reste néanmoins nécessaire pour élucider le chemin réactionnel emprunté par chaque molécule. À ce titre, la résonance magnétique nucléaire associée à la modélisation moléculaire pourrait constituer des outils d'investiga- tion particulièrement pertinents pour caractériser les formes oxydés des acides et des esters. Bien que la méthode DPPH fournisse de précieuses informations quant à la réactivité intrinsèque de composés phénoliques vis-à-vis du radical DPPH, elle présente néanmoins l'inconvénient de ne pas mettre en compétition l'antioxydant avec un substrat oxydable. Cet aspect est en effet discutable, tant il est vrai que la seule réduction d'espèces oxydantes n'est pas suffisante pour contrer l'oxydation. À cet égard, seul un piégeage rapide des radicaux libres à proximité des substrats cibles de l'oxydation permettrait de protéger ces derniers. Pour prendre en compte un tel phénomène, une approche compétitive impliquant I'utilisation d'un substrat est par conséquent nécessaire. Par ailleurs, en parallèle de ces mécanismes de compétition, le type de système employé selon qu'il soit homogène ou hétérogène peut moduler la capacité antioxydante d'un composé donné. Dans ce contexte, l'étude en milieu hétérogène des propriétés antioxydantes d'acides phénoliques libres ou estérifiés nous a semblé particulièrement pertinente. Aussi avons-nous employé la méthodologie CAT (conjugated autoxidable trienes) mise en œuvre en milieu émulsifié pour étudier l'influence de la longueur de la chaîne alkyle sur le comportement antioxydatif de composés phénoliques. Les résultats obtenus pour une série d'homologues lipophiles d'acides chlorogénique et rosmarinique ont montré que l'augmentation de la longueur de chaîne aliphatique ne conduisait pas forcément à une amélioration de la capacité antioxydante comme le prédit le « Paradoxe Polaire ». En effet, un phénomène de rupture («cut-off ») de la capacité antioxydante pour des longueurs de chaîne de 12 et 8 atomes de carbone a été observé pour les dérivés lipophiles des acides chlorogénique et rosmarinique respectivement.

En faisant l'hypothèse que la localisation de I'antioxydant est de prime importance quant à l'expression de son activité antioxydante, nous nous sommes intéressés en second lieu à la répartition de l'antioxydant dans le système réactionnel et plus particulièrement dans la phase continue (aqueuse) de l'émulsion. Dans cette phase, la teneur relative (en \%) de l'antioxydant a pu être déterminée et a servi de descripteur.

Concernant les esters d'acide chlorogénique, l'évolution de ce descripteur en fonction du nombre d'atomes de carbone de la chaîne alkyle a également été caractérisée par un phénomène de « cut-off », centré sur 12 atomes de carbone. Ce résultat pourrait expliquer la meilleure efficacité antioxydante du chlorogénate de dodécyle. Cependant, la faible différence de distribution entre les chlorogenates d'octyle et dodécyle ne concorde pas avec la forte 
différence d'activité antioxydante entre ces dernières. Ainsi, puisque l'allure cinétique des courbes $d$ 'antioxydation suggèrent fortement que le phénomène de cut-off provient des produits d'oxydation et non des molécules telles qu'introduite initialement dans le test, nous avons postulé que la localisation des produits d'oxydation pouvait être un facteur déterminant sur la valeur finale de la capacité antioxydante. La mise en évidence de ce type de contribution pourrait constituer une perspective de travail intéressante. Concernant les esters d'acide rosmarinique, il est apparu que le descripteur employé (pourcentage d'antioxydant dans la phase aqueuse) n'était pas suffisant pour expliquer le comportement antioxydant observé. Ce descripteur, en effet, ne renseigne pas sur les quantités et l'organisation des molécules d'antioxydant présentes à I'interface et/ou dans la phase huileuse ce qui, dans le cas de l'acide rosmarinique et ses esters, semble être la principale cause de l'effet « cutoff » observé. L'ensemble de ces résultats montre qu'en milieu hétérogène, la connaissance de la répartition de l'antioxydant et/ou de ses formes oxydées dans les différentes phases du système, est de prime importance quant à la compréhension de son activité et de ces modes d'action. À l'avenir, l'isolement et la caractérisation des différents produits d'oxydation, en relation avec des études de distribution, pourrait permettre de rationaliser les phénomènes oxydatif et antioxydatif à l'œuvre dans les systèmes hétérogènes.

Finalement, les résultats obtenus et les interprétations qui en découlent, pourraient constituer le point de départ d'une étude de la lipophilisation d'extraits végétaux riches en acides chlorogénique et rosmarinique ainsi que l'évaluation du pouvoir antioxydant des molécules formées après réaction. En outre, l'amélioration des connaissances apportée par ce travail et les études à venir, ainsi que la demande croissante de molécules actives amphiphiles dans les industries pharmaceutiques et cosmétiques, devrait à terme, contribuer à une valorisation plus importante de ces composés et de leurs sources végétales.

\section{PUBLICATIONS ISSUES DE CE TRAVAIL}

Lopez Giraldo LJ, Laguerre M, Lecomte J, FigueroaEspinoza MC, Pina M, Villeneuve P. Lipophilisation de composés phénoliques par voie enzymatique et propriétés antioxydantes des molécules lipophilisées. OCL 2007 ; 14 : 51-9.

Lopez Giraldo LJ, Laguerre M, Lecomte J, et al. Lipasecatalyzed synthesis of chlorogenate fatty esters in solvent-free medium. Enz Microb Tech 2007 ; 41 : 721-6.
Yuji H, Weiss J, Villeneuve P, Lopez Giraldo L), Figueroa-Espinoza MC, Decker EA. Ability of surface active antioxidants to inhibit lipid oxidation in oil-in-water emulsion. J Agric Food Chem 2007 ; 55 : 11052-6.

Laguerre M, Lopez-Giraldo LI, Lecomte J, Pina M, Villeneuve P. Outils d'évaluation in vitro de la capacité antioxydante. OCL 2007; 14: 278-92.

Laguerre M, López-Giraldo LJ, Lecomte J, et al. Conjugated autoxidizable triene (CAT) assay: a novel spectrophotometric method for determination of antioxidant capacity using triacylglycerol as ultraviolet probe. Anal Biochem 2008; 380 : 282-90.

López-Giraldo L), Laguerre M, Lecomte J, et al. Kinetic and stoichiometry of the reaction of chlorogenic acid and its alkyl esters against the DPPH radical. J Agric Food Chem 2009 ; 57 : 863-70.

Laguerre M, Lopez-Giraldo LJ, Lecomte J, Villeneuve P. Widespread methods and new analytical approaches in antioxidant evaluation. Inform $2009 ; 20: 328-32$.

Laguerre M, López Giraldo L), Piombo G, et al. Characterization of olive leaf phenolics by ESI-MS and evaluation of their antioxidant capacities by the CAT assay. Am Oil Chem Soc 2009 (sous presse). 